\title{
Paresthesia of nerve due to acute apical periodontitis of a non-vital tooth: report of two cases
}

\author{
Maryam Mojtahed bidabadi ${ }^{1}$, Hengameh Ashraf ${ }^{2}$, Pejman Arjmand inaloo ${ }^{3}$, Soheila Darmiani ${ }^{1 *}$ \\ ${ }^{1}$ Postgraduate Student of Endodontics, School of Dentistry, Shahid Beheshti University of Medical Sciences, Tehran, Iran \\ ${ }^{2}$ Professor of Endodontics Department, School of Dentistry, Shahid Beheshti University of Medical Sciences, Tehran, Iran \\ ${ }^{3}$ Dentist \\ *Corresponding author E-mail: soheiladarmiani@yahoo.com
}

\begin{abstract}
Paresthesia or dysesthesia of the inferior alveolar nerve (IAN) as the result of endodontic treatment or periodontal disease is a rare but serious occurrence. This paper describes two cases of paresthesia and dysesthesia of the IAN due to acute apical periodontitis of a right mandibular second molar and a right mandibular second premolar in proximity to inferior alveolar canal. Symptoms of sensory disruption disappeared approximately 2 months after conventional endodontic treatment associated with antibiotic therapy. One year later both teeth were still asymptomatic.
\end{abstract}

Keywords: Endodontic Pain, Inferior Alveolar Nerve, Injuries, Paresthesia.

\section{Introduction}

Paresthesia is defined as an abnormal sensation caused by chemical, mechanical or thermal nerve injury (Yeler et al. 2004). Patients describe it as burning, aching, prickling, tingling, pins and needles and numbness (Zmener 2004).

Sensory disturbances in the area innervated by the inferior alveolar nerve and its branches, namely the chin, lower lip and oral mucosa are infrequent but serious complication in routine dental practice (Zmener 2004, Gallas-Torreire et al. 2003). Numerous factors such as trauma, infectious diseases, demineralization, idiopathic conditions and connective tissue diseases may be responsible for IAN paresthesia (Gallas-Torreire et al. 2003). Neural injury may occur as the result of mechanical (compression), chemical or thermal factors (Yeler et al. 2004, Ograyd 1996, Goldberg \& Galbraith 1984, Giuliani 2001). Local factors responsible include endodontic and surgical factors. Severe periapical infections, iatrogenic sequel of RCT, overfilling, toxicity of the filling materials and sealers, over-extension of endodontic files and broken instruments fall into the category of endodontic etiologies of IAN paresthesia (Abbott 1997, Poveda et al. 2006, Di Lenarda et al.2000). Fracture of the mandible, improper implant placement, nerve compression as the result of surgical instrumentation and orthognathic surgeries are among the surgical factors that can lead to sensory disturbances of the IAN (Di Lenarda et al.2000, Kafas et al.2009, Ozkan et al.2008). Paresthesia due to the presence of an infected impacted tooth has also been reported (Kafas ET al.2009).

Mandibular second molar and premolar teeth are most commonly affected (Pogrel 2007). Examination of the affected area can be done using thermal, mechanical, electrical or chemical tests provoking subjective responses (Mohammadi 2010).

Paresthesia due to periapical lesions is attributed to mechanical compression of the nerve and ischemia associated with the inflammatory process (edema), local pressure on the mental nerve resulting from accumulation of purulent exudate in the mandibular bone as well as the toxic metabolic products of bacteria or the inflammatory products released as the result of tissue injury (Giuliani et al.2001, Morse 1997, Ellinston \& Hoen 1996).

This article describes the diagnosis and management of two cases of IAN paresthesia due to endodontic infection and subsequent acute apical periodontitis.

Case 1:

A twenty-nine year-old female patient was referred to our clinic complaining of numbness at the right side of her lower lip and strong pain at the mandibular right second molar tooth. The patient was healthy with no underlying systemic condition. She was examined clinically and radiographically(Fig.1). Root canal therapy was performed for the respective tooth. After the administration of local anesthesia and rubber dam placement, caries excavation was done followed by access cavity preparation. Working length was determined using No. $15 \mathrm{~K}$ file (Dentsply, Maillefer) which was then confirmed using Ipex electronic apex locator (NSK, Japan). The root canals were instrumented using ProTaper (Dentsply-Maillefer, Ballaigues, Switzerland) rotary instruments and RC Prep (Premier, Norristown, PA, USA) as a lubricant. The final preparation file was Pro-Taper F3. Copious irrigation was done with $2.5 \%$ sodium hypochlorite solution during the procedure. Care was taken not to push the irrigating solution beyond the apex to prevent any further damage to the nerve. Canals were dried with paper points and dressed with calcium hydroxide paste. After 10 days, obturation of root canals was performed with gutta percha points using lateral condensation technique and AH26 sealer (Dentsply-Maillefer, Ballaigues, Switzerland). Analgesics and 20 tablets of V-penicillin $(500 \mathrm{mg}$ ) were prescribed for the patient during the root canal therapy period. The patient reported improvement in pain and burning sensation two months after the treatment. One year later, she was still asymptomatic. 


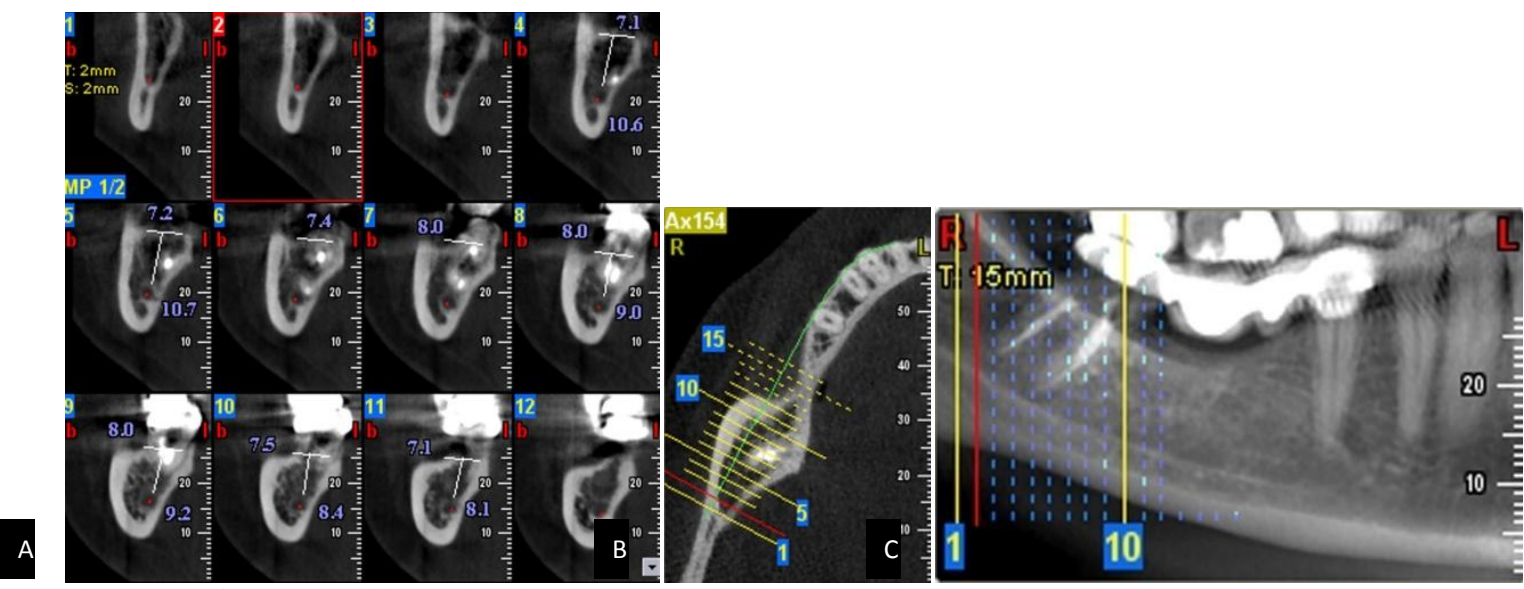

Fig. 1: Dental CT Scan of a 29 Year-Old Female Patient, Demonstrating the Mandibular Second Molar in Close Proximity to the Inferior Alveolar Canal. Approximation Is Located at the Distal Root and Vertical Lines are Superimposed Reflecting the Tomographical slices along the Perpendicular Plane (A). Details of Slices 1 To 10 Disclose Proximity to Inferior Alveolar Canal (B, C). The Patient Has Paresthesia.

\section{Case 2:}

A 21 year-old man presented to the Endodontics Department of Shahid Beheshti University complaining of pain in his right mandibular second premolar tooth. The patient had no history of alcohol consumption or tobacco use and mentioned no personal or family history of systemic disease. Intraoral examinations showed an extensive carious lesion at the distal surface of tooth \#29. Vitality tests revealed pulp necrosis. Periapical radiograph were obtained at the first session revealing acute periapical periodontitis and close proximity of the root apex to the mental foramen. No significant apical radiolucency was observed. The patient reported severe pain at the location of the respective tooth along with paresthesia at the right corner of the lower lip gradually extending posteriorly and towards the chin. Intraoral clinical examination showed that the respective tooth and the labial gingiva were tender and sensitive to palpation. Based on the patient's symptoms and the close proximity of the tooth apex to the mental foramen, Cone beam computed tomography (CBCT) was requested to determine the anatomical relationship and distance between the apex and mental foramen. CBCT confirmed the close proximity (Fig.2).
The tooth was anesthetized by a mental injection and isolated. Access cavity was prepared. Working length was determined using an apex locator (Raypex 5, Dentsply, Maillefer, Ballaigues, Switzerland) and confirmed with PA radiography. Root canal instrumentation was done using ProTaper rotary files (Dentsply, Maillefer, Ballaigues, Switzerland) up to F3. Apical preparation was done using \#30 and \#35 SS files (Dentsply, Maillefer, Ballaigues, Switzerland). During preparation, canal irrigation was performed using $0.5 \%$ sodium hypochlorite solution. Utmost care was taken not to push the irrigation solution through the apex. Over-instrumentation was avoided as well. $\mathrm{Ca}(\mathrm{OH}) 2$ was applied as an intracanal dressing for 7 days. In the next session, canals were filled with gutta percha and $\mathrm{AH} 26$ sealer. Care was taken not to push the sealer through the apex into the periapical tissue. Access cavity was temporarily restored with Coltosol. The patient was referred to the Department of Restorative Dentistry for final restoration. At one week follow up, the patient had no pain or paresthesia and the respective tooth was asymptomatic. At the second follow up one year later, the patient had no problem with the tooth.

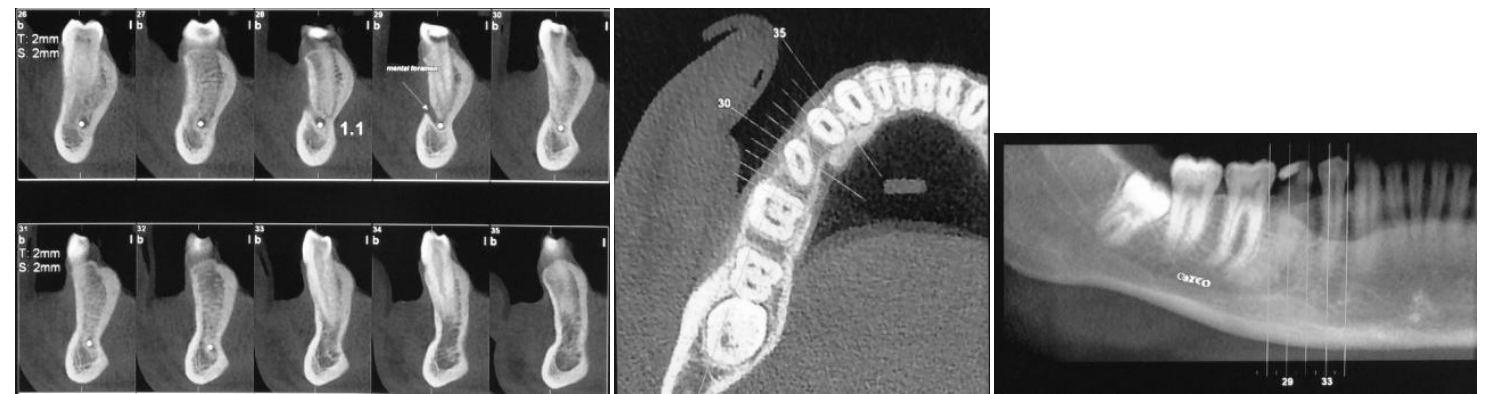

Fig. 2: Dental CT Scan of a 21 Year-Old Male Patient, Demonstrating the Mandibular Second Premolar in Close Proximity to the Mental Foramen. (A). Details of Slices Disclose Proximity to Mental Foramen (B, C). The Patient Has Paresthesia.

\section{Discussion}

According to Seddon's classification for peripheral nerve injury, there are three levels of injuries based on the severity, prognosis and time to recovery: Neuropraxia, Axonotmesis and Neurotmesis (Seddon 1943). Neuropraxia is a temporary conduction block after mild compression of the nerve trunk (i.e. paresthesia or dysesthesia of the lip and chin region in case of IAN /mental nerve injury). Full recovery occurs after a few hours to days. Loss of axonal continuity occurs in Axonotmesis. Partial recovery takes a couple of months. Neurotmesis is the most severe type of nerve injury (Seddon 1943, Becelli et al. 2002).

Examination of the affected area can be carried out by using thermal, mechanical, electrical or chemical tests that elicit subjective responses (Mohammadi 2010). CBCT is an excellent preoperative diagnostic tool for visualization of the mandibular canal (Burstein et al. 2008).

The molar roots area and the location of the IAN can be estimated in the buccolingual plane without the superimposition of the surrounding anatomical structures (Kim et al. 2010).

In a study evaluating the spatial relationship between the posterior teeth and the mandibular canal in 22 mature dried human skulls accounting for 26 specimen sections, second premolars and second molars had the closest distance to the canal with a mean distance of 4.7 and $3.7 \mathrm{~mm}$, respectively. The mandibular canal was located directly beneath the root apices of the posterior teeth in 5\% of cases. Data also showed that by decreasing the mandibular height, the distance between the canal and root apices decreased as well (Denio et al. 1992). In the mandibular second molar area, the distance from apices to IAN pedicle is usually less 
than $1 \mathrm{~mm}$. This distance ranges from 1 to $4 \mathrm{~mm}$ for the first molar (Tilotta-Yasukawa et al. 2006).

Adiguzel et al. evaluated whether age and gender differences were predictive of inferior alveolar nerve position with respect to mandibular first molar roots and found that nerve- root apex distances were significantly shorter in males and females aged 16-25 and 56-65 years than in other age groups (Adiguzel et al. 2012). In a study by Givol et al and some others most patients complaining of the paresthesia of the IAN were females (Givol et al. 2011, Givol et al. 2010, Bjørndal \& Reit 2008, Givol et al. 2002).

Literature regarding the IANP and MNP due to periapical conditions is scarce (Gvotz et al. 1998, von ohle \& El ayouti 2010).Givol in his study reported that of 262 cases of sensory disturbances, $16(6.1 \%)$ occurred after endodontic treatment and $68.8 \%$ of them occurred after endodontic treatment of mandibular second molar tooth (Givol et al. 2011). Rate of sensory disturbances following endodontic treatment was $5.8 \%$ in a study by Garisto et al. (Garisto et al. 2010). Scolozzi reported 4 cases of sensory disturbances following endodontic therapy of mandibular molars. They had to perform a sagittal osteotomy to resect the endodontic paste and decompress the nerve; which led to immediate relief of pain in patients (Scolozzi et al. 2004).

A consensus has been reached that toxic metabolites released by the activity of intracanal microorganisms and the inflammatory products are responsible for sensory disturbances of the IAN or mental nerve in some endodontic patients (Yeler et al. 2004, Di Lenarda et al. 2000).

Clinicians should be well aware of this condition and know that it is usually resolved with efficient endodontic treatment and associated antibiotic therapy (Ozkan et al. 2008, Mohammadi 2010). Also, it should be noted that the longer the neural irritation, the greater the magnitude of nerve degeneration and the higher the risk of permanent paresthesia (Tilotta-Yasukawa et al. 2006).

\section{References}

[1] Yeler H, Ozeç I, Kiliç E (2004), Infection-related inferior alveolar and mental nerve paresthesia: case reports. Quintessence Int 35(4), 313-6.

[2] Zmener O (2004), mental nerve paresthesia associated with an adhesiv e resin restoration: A case report. J Endod 30, 117-9.

[3] Gallas-Torreire MM, Reboiras-Lopez MD, Garcia-Garcia A, GandaraRey J (2003), Mandibular nerve paresthesia caused by endodontic trea tment .Med Oral 8, 299-303.

[4] Ograyd JF (1996), mental paresthesia: An ominous symptom. Case re ports. Aus Dent J 41,370-2.

[5] Goldberg MH, Galbraith DA (1984), late onset of Mandibular and ling ual dysesthesia secondary to postextraction infection. Oral Surg 58, 26 9-271.

[6] Giuliani M, Lajolo C, Deli G, Silveri C (2001), Inferior alveolar nerve paresthesia caused by endodontic pathosis: A case report and review of the literature. Oral Surg Oral Med Oral Pathol 92, 670-4.

[7] Abbott PV (1997), Lower lip paresthesia following restoration of a sec ond premolar tooth: case report. Aust Dent J 42, 297-301.

[8] Poveda R, Bagán JV, Fernández JM, Sanchis JM (2006), mental nerve paresthesia associated with endodontic paste within the mandibular ca nal: report of a case. Oral Surg Oral Med Oral Pathol Oral Radiol End od 102(5), e46-9. Epub Sep 12.

[9] Di Lenarda R, Cadenaro M, Stacchi C (2000), Paresthesia of the ment al nerve induced by periapical infection: a case report. Oral Surg Oral Med Oral Pathol Oral Radiol Endod 90(6), 746-9.

[10]Kafas P, Upile T, Angouridakis N, Stavrianos C, Dabarakis N, Jerjes W (2009), Dysaesthesia in the mental nerve distribution triggered by a foreign body: a case report. Cases J 28, 2:169. doi: 10.1186/1757-162 6-2-169.

[11]Ozkan BT, Celik S, Durmus E (2008), Paresthesia of the mental nerve stem from periapical infection of mandibular canine tooth: a case rep ort. OralnSurg Oral Med Oral Pathol Oral RadiolEndod 105(5), 28-31.

[12]Pogrel MA (2007), Damage to the inferior alveolar nerve as the result of root canal therapy. J Am Dent Assoc 138, 65-9.

[13]Mohammadi Z (2010), Endodontics-related paresthesia of the mental and inferior alveolar nerves: an updated review. J Can Dent Assoc 76, a117.

[14]Morse DR (1997), Infection-related mental and inferior alveolar nerve paresthesia: literature review and presentation of two cases. J Endod 2 3, 457-460.
[15]Ellinston NK, Hoen MM (1996), Infectious transient dental paresthesi a Gen Dent 44, 66-9.

[16] Seddon HI (1943), three types of nerve injury.Brain 66, 237-288.

[17]Becelli R, Renzi G, Carboni G (2002), Inferior alveolar nerve impairm ent after mandibular sagittal split osteotomy. An analysis of spontaneo us recovery patterns observed in 60 patients. J Cranio of ac Surg 13, 3 15-320.

[18]Burstein J, Mastin C, Le B (2008), a voiding injury to the inferior alve olar nerve by routine use of interaoperative radiographs during implan t placement. J Oral Implant 34, 34-8.

[19] Kim TS, Caruso JM, Christensen H, Torabinejad M (2010), A compar ison of cone beam computed tomography and direct measurement in $t$ he examination of the mandibular canal and adjacent structures. J End od 36, 1191-4.

[20]Denio D, Torabinejad M, Bakland LK (1992), Anatomical relationshi $\mathrm{p}$ of themandibular canal to its surrounding structure in mature mandib les .J Endod 18, 161-5.

[21]Tilotta-Yasukawa F, Millot S, El Haddioui A, Bravetti P, Gaudy JF (2 006), Labiomandibular paresthesia caused by endodontic treatment: an anatomic and clinical study. Oral Surg Oral Med Oral Pathol Oral Ra diol Endod 102, e47-59.

[22] Adiguzel O, Yigit-Ozer S, Kayal S, Akkus Z (2012), Patient-specific f actors in the proximity of the inferior alveolar nerve to the tooth apex . Med Oral Patol Oral Cir Bucal 1, 17(6):e1103-8.

[23]Givol N, Rosen E, Bjørndal L, Taschieri S, Ofec R, Tsesis I (2011), M edico-legal aspects of altered sensation following endodontic treatmen t: a retrospective case series. Oral Surg Oral Med Oral Pathol Oral Ra diol Endod 112(1), 126-31. doi: 10.1016/j.tripleo.2011.01.007. Epub 2 011 Mar 31.

[24] Givol N, Rosen E, Taicher S, Tsesis I (2010), Risk management in en dodontics. J Endod 36, 982-4

[25]Bjørndal L, Reit C (2008), Endodontic malpractice claims in Denmark 1995-2004. Int Endod J 41, 1059-65

[26] Givol N, Taicher S, Halamish-Shani T, Chaushu G (2002), Risk mana gement aspects of implant dentistry. Int J Oral Maxillofac Implants 17 ,258-62.

[27] Gvotz KA, Al-Nawas B, de Aguiar EG, Schulz A, Wagner W (1998), Treatment of injuries to the inferior nerve after endodontic procedures Clin Oral Investig 2,73-76.

[28] von ohle C, El ayouti A (2010), Neurosensory impairment of the me ntal nerve as a sequel of periapical periodontitis.case report and revie w. Oral Surg Oral Med Oral Pathol Oral RadiolEndod 110, 84-89.

[29] Garisto GA, Gaffen AS, Lawrence HP, Tenenbaum HC, Haas DA (20 10), Occurrence of paresthesia after dental local anesthetic administra tion in the United States. J Am Dent Assoc 141, 836-44.

[30]Scolozzi P, Lombardi T, Jaques B (2004), Successful inferior alveolar nerve decompression for dysesthesia following endodontic treatment: report of 4 cases treated by mandibular sagittal osteotomy. Oral Surg Oral Med Oral Pathol Oral Radiol Endod 97(5), 625-31. 